\title{
Hemodynamic Changes Caused by Multiple Stenting in Vertebral Artery Fusiform Aneurysms: A Patient-Specific Computational Fluid Dynamics Study
}

\author{
(D) N. Lv, DW. Cao, DI. Larrabide, (D). Karmonik, DD. Zhu, (D). Liu, (D). Huang, and DY. Fang
}

\begin{abstract}
BACKGROUND AND PURPOSE: The multiple stent placement technique has largely improved the long-term outcomes of intracranial fusiform aneurysms, but the hemodynamic mechanisms remain unclear. In this study, we analyzed the hemodynamic changes caused by different stent-placement strategies in patient-specific models using the computational fluid dynamics technique, aiming to provide evidence for clinical decision-making.
\end{abstract}

MATERIALS AND METHODS: Ten vertebral artery fusiform aneurysms were included, and their patient-specific computational fluid dynamics models were reconstructed. A fast virtual stent placement technique was used to simulate sequential multiple stent placements (from a single stent to triple stents) in the vertebral artery fusiform aneurysm models. Hemodynamic parameters, including wall shear stress, pressure, oscillatory shear index, relative residence time, and flow pattern, were calculated and compared among groups with different numbers of stents.

RESULTS: Virtual stents were deployed in all 10 cases successfully, consistent with the real stent configuration. Wall shear stress decreased progressively by $7.2 \%, 20.6 \%$, and $25.8 \%$ as the number of stents increased. Meanwhile, relative residence time and pressure increased on average by $11.3 \%, 15.4 \%$, and $45.0 \%$ and by $15.7 \%, 21.5 \%$, and $28.2 \%$. The oscillatory shear index showed no stable variation trend. Flow patterns improved by weakening the intensity of the vortices and displacing the vortex center from the aneurysmal wall.

CONCLUSIONS: Stent placement modifies hemodynamic patterns in vertebral artery fusiform aneurysms, which might favor thrombosis formation in the aneurysmal sac. This effect is amplified with the number of stents deployed. However, a potential risk of rupture or recanalization exists and should be considered when planning to use the multiple stent placement technique in vertebral artery fusiform aneurysms.

ABBREVIATIONS: CFD = computational fluid dynamics; OSI = oscillatory shear index; RRT = relative residence time; VAFA = vertebral artery fusiform aneurysm; WSS $=$ wall shear stress

$V^{2}$ ertebral artery fusiform aneurysm (VAFA) is the most frequent type of intracranial fusiform aneurysm, which is often associated with nerve compression or ischemia, but bleeding is

Received February 14, 2017; accepted after revision August 4.

From the Department of Neurosurgery (N.V., W.C., D.Z., Y.F., Q.H., J.L.), Changhai Hospital, Second Military Medical University, Shanghai, China; National Scientific and Technical Research Council of Argentina (I.L.), Buenos Aires, Argentina; and MRI Core (C.K.), Houston Methodist Research Institute, Houston, Texas.

Nan Lv and Wei Cao contributed equally to this work and should be regarded as co-first authors.

This work was supported by the National Natural Science Foundation of China (grant Nos. 81571118 and 81301004), the National Research and Development Project of Key Chronic Diseases (grant No. 2016YFC1300700), and National Scientific and Technical Research Council of Argentina (grant Nos. PICT 2014-1730 and PICT 2015-0006).

Please address correspondence to Qinghai Huang, MD, and Yibin Fang, MD, Department of Neurosurgery, Changhai Hospital, Changhai Rd 168, Shanghai, 200433, China; e-mail: ocinhqh@163.com; fangyibin@vip.163.com

- Indicates open access to non-subscribers at www.ajnr.org

http://dx.doi.org/10.3174/ajnr.A5452 relatively rare. ${ }^{1,2}$ However, in case of rupture, the mortality and early rebleeding rate of fusiform aneurysms are even higher than for saccular aneurysms. ${ }^{3}$ Since the development of neuroimaging techniques and interventional devices, endovascular treatment has been the primary method for VAFAs. Although flow diverters have shown their effectiveness in treating aneurysms, the risk of ischemic events in posterior circulation cannot be ignored, especially when vital branches are covered. ${ }^{4}$ Therefore, stepwise overlapping stent placement is still a safer and more effective choice for VAFAs than flow diverters, and the long-term improvement has been demonstrated. ${ }^{5}$

Hemodynamic studies of saccular aneurysms have revealed that stent placement can reduce the impinge flow and wall shear stress (WSS); this effect may favor thrombus formation and consequently cure the aneurysm. ${ }^{6}$ However, the morphology of fusiform aneurysms is entirely different from that of saccular aneurysms; this difference may result in unique flow patterns before and after stent placement. Most important, gaining knowledge of 
Table 1: Clinical data of 10 patients with VAFAs

\begin{tabular}{|c|c|c|c|c|c|}
\hline Patient No. & Age (yr) & Sex & Ruptured & Treatment & Follow-Up \\
\hline Case 1 & 46 & Male & No & $3 \mathrm{EP}$ & $8 \mathrm{mo}$, cured \\
\hline Case 2 & 53 & Male & No & $2 \mathrm{EP}$ & $12 \mathrm{mo}$, thrombosis formation \\
\hline Case 3 & 39 & Female & Yes & $2 \mathrm{EP}+$ coils & Withdraw \\
\hline Case 4 & 37 & Male & Yes & $2 \mathrm{EP}+$ coils & $6 \mathrm{mo}$, cured \\
\hline Case 5 & 51 & Male & No & $2 \mathrm{EP}+$ coils & $7 \mathrm{mo}$, thrombosis formation \\
\hline Case 6 & 59 & Male & No & $3 \mathrm{EP}$ & $13 \mathrm{mo}$, cured \\
\hline Case 7 & 49 & Female & Yes & $2 \mathrm{EP}+$ coils & $12 \mathrm{mo}$, cured \\
\hline Case 8 & 46 & Male & Yes & $3 \mathrm{EP}+$ coils & 12 mo, cured \\
\hline Case 9 & 60 & Male & No & $2 \mathrm{EP}$ & $6 \mathrm{mo}$, stable \\
\hline Casel0 & 46 & Male & No & $2 \mathrm{EP}$ & $12 \mathrm{mo}$, thrombosis formation \\
\hline
\end{tabular}

Note:-EP indicates Enterprise self-expanding stent.

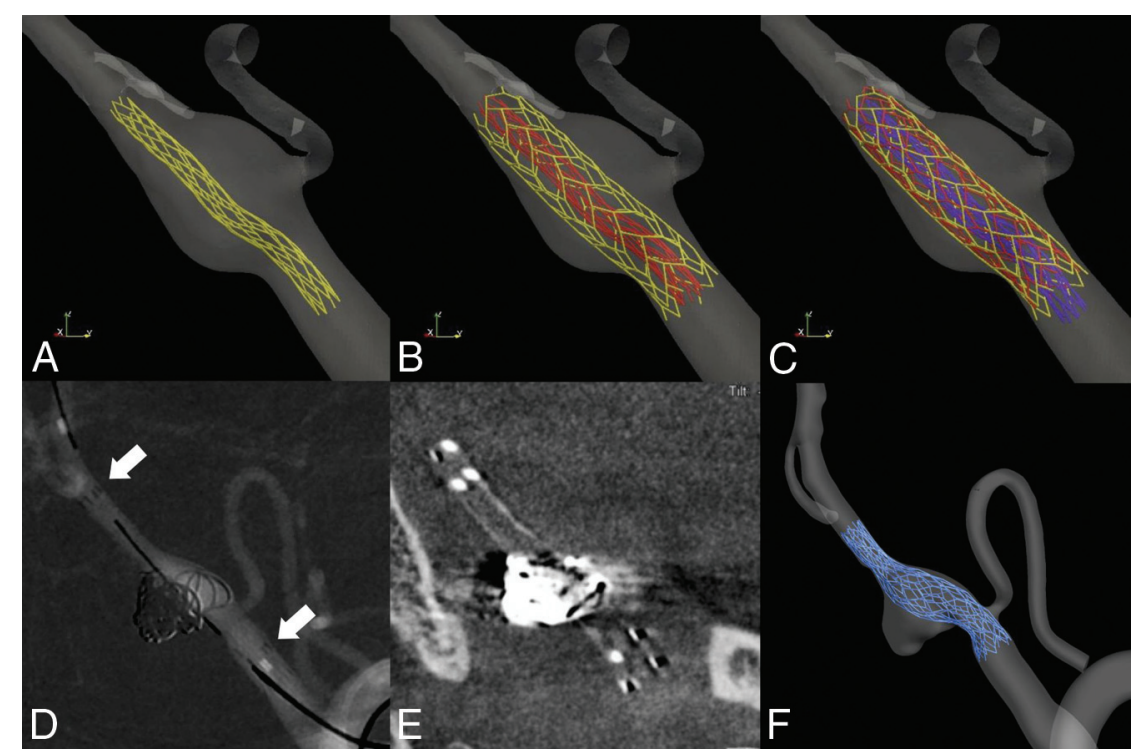

FIG 1. Process of virtual stent deployment. A-C, Three virtual Enterprise stents were deployed one by one. $D$ and $E$, Location of real stents in 2D angiography and DynaCT (Siemens). F, Virtual stents deployed in a similar location with the real counterparts.

how intra-aneurysmal flow patterns change when the number of overlapping stents increases is of great importance because it may provide some evidence for optimizing stent-placement strategies.

In this study, we compared the hemodynamic changes caused by an increasing number of stents in patient-specific cases with measured boundary conditions with the computational fluid dynamics (CFD) technique, aiming to provide evidence for clinical decision-making for fusiform aneurysms.

\section{MATERIALS AND METHODS}

The institutional review board of the Second Military Medical University-affiliated Changhai Hospital approved this retrospective study, and the requirement for informed consent was waived. In addition, we have not conducted research outside our country of residence.

\section{Patients and Imaging Acquisition}

From January 2015 to December 2015, nineteen VAFAs in 19 patients were diagnosed in our hospital by $3 \mathrm{D}$ rotational angiography. Four patients were excluded because the lesion involved the extracranial V3 portion or basilar artery; and 5 patients were excluded because a double lumen sign was observed by DSA. Finally, 10 patients (male/female ratio $=8: 2$; mean age, 48.6 years) with 4 ruptured and 6 unruptured VAFAs were included in the study. The clinical data of the patients are shown in Table 1.

$3 \mathrm{D}$ rotational angiography was performed with the Artis zee biplane angiographic system (Siemens, Erlangen, Germany). A 5-second DSA acquisition protocol was adopted, and $18 \mathrm{~mL}$ of contrast agent was injected through the vertebral artery at a rate of $3 \mathrm{~mL} / \mathrm{s}$. During the 5-second acquisition after a 1 -second delay, a $200^{\circ}$ rotation of the C-arm was performed to obtain 133 frames. All acquired 5-second DSA data were transferred to the syngo X Workplace (Siemens) for reconstruction of the $3 \mathrm{D}$ artery vessel tree and exported into a stereolithography format.

\section{Models of VAFAs and Stents}

The stereolithography models were segmented and smoothed by Geomagic Studio 9.0 software (Geomagic, Morrisville, North Carolina) and then imported into ICEM CFD 11.0 (ANSYS, Canonsburg, Pennsylvania) to create volume grids for the CFD simulations. The vessel wall was divided into 3 parts: aneurysm, parent artery, and other vessels.

The virtual stent model simulated the Enterprise self-expanding stent (Codman \& Shurtleff, Raynham, Massachusetts), which is one of the most widely used commercial self-expanding stents. Three different stent sizes were constructed, including $4.5 \mathrm{~mm}$ (diameter) $/ 22 \mathrm{~mm}$ (length), $4.5 / 28 \mathrm{~mm}$, and $4.5 / 37 \mathrm{~mm}$. The porosity and metalcoverage rates were $94 \%$ and $6 \%$, respectively. The stents were reconstructed using the fast virtual stent method and deployed in the VAFA models as previously described by Larrabide et al. ${ }^{7}$ The proximal and distal ends of the virtual stents were determined according to the realistic locations on $2 \mathrm{D}$ angiographic imaging of each patient. We simulated 3 scenarios: single stent, 2 overlapping stents, and 3 overlapping stents, as well as the prestenting status (Fig 1).

\section{CFD Analysis and Hemodynamic Parameters}

CFD simulations were performed by CFX 11.0 (ANSYS). The vessel was considered a rigid wall with no-slip boundary conditions. The governing equations underlying the calculation were the Navier-Stokes formulations, with an assumption of a laminar and incompressible blood flow (density, $\rho=1050 \mathrm{~kg} / \mathrm{m}^{3}$; dynamic viscosity, $\mu=0.00345 \mathrm{~Pa} \times \mathrm{s}$ ). Inlet boundary conditions for all scenarios were imposed by a pulsatile velocity waveform obtained from transcranial Doppler sonography, and to account for the effect of autoregulation of flow in the brain, we defined the outlet 

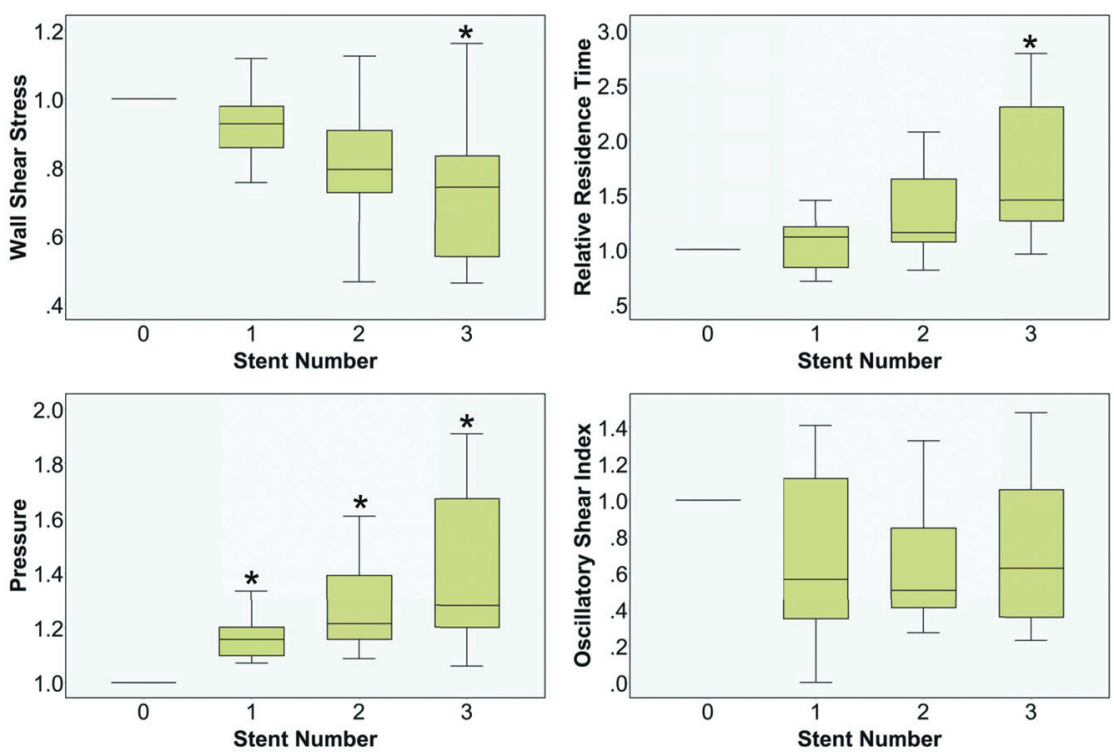

FIG 2. Box figure of hemodynamic analysis results. The asterisk indicates that the parameter was significantly different from the prestenting status.

Table 2: Hemodynamic parameters of VAFAs with different stenting strategies ${ }^{a}$

\begin{tabular}{lccccc}
\hline \multicolumn{1}{c}{ Parameters } & 0 Stent & 1 Stent & 2 Stents & 3 Stents & P Value \\
\hline Wall shear stress $(\%)(n=10)$ & 100 & 92.8 & 79.4 & 74.2 & .003 \\
Oscillatory shear index $(\%)(n=10)$ & 100 & 56.5 & 50.5 & 62.6 & .169 \\
Pressure $(\%)(n=10)$ & 100 & 115.7 & 121.5 & 128.2 & $<.001$ \\
Relative residence time (\%) $(n=10)$ & 100 & 111.3 & 115.4 & 145.0 & .004 \\
\hline
\end{tabular}

${ }^{a}$ Data are expressed as the median of the variation rate.

Table 3: $P$ values of multiple comparisons among different stenting strategies

\begin{tabular}{lccc}
\multicolumn{1}{c}{ Parameters } & $\begin{array}{c}\text { 0 Stent vs } \\
\text { 1 Stent }\end{array}$ & $\begin{array}{c}\text { 0 Stent vs } \\
\text { 2 Stents }\end{array}$ & $\begin{array}{c}\text { 0 Stent vs } \\
\text { 3 Stents }\end{array}$ \\
\hline WSS $(n=10)$ & .498 & .063 & .007 \\
Pressure $(n=10)$ & .037 & .001 & $<.001$ \\
RRT $(n=10)$ & .904 & .251 & .009 \\
\hline
\end{tabular}

as the opening boundary condition with zero static pressure, assuming no downstream hemodynamic resistance. The cardiac cycle of 0.8 seconds was discretized at a time-step of 0.001 seconds for the numeric simulations. For each model, we simulated 3 continuous cardiac cycles to ensure the numeric stability of the simulation, and results are reported from the last cycle. The results were postprocessed and visualized with CFX 11.0.

Several hemodynamic parameters were calculated in this study: normalized wall shear stress, pressure of the aneurysmal wall, oscillatory shear index (OSI), and relative residence time (RRT). The time-averaged WSS was further averaged over the dome area (the entire luminal surface of the aneurysm sac) and then normalized by the average parent vessel WSS in the same patient to allow comparison among different patients. OSI, a nondimensional parameter, measures the directional change of WSS during the cardiac cycle. RRT, a combination of WSS and OSI, reflects the residence time of blood near the wall:

$$
W S S=\frac{1}{\mathrm{~T}} \int_{0}^{\mathrm{T}}\left|W S S_{\mathrm{i}}\right| d t,
$$

2)

$$
O S I=\frac{1}{2}\left\{1-\frac{\left|\int_{0}^{\mathrm{T}} W S S_{\mathrm{i}} d t\right|}{\int_{0}^{\mathrm{T}}\left|W S S_{\mathrm{i}}\right| d t}\right\}
$$

3)

$$
\begin{array}{r}
R R T=\frac{1}{(1-2 \times O S I) \times W S S} \\
=\frac{1}{\frac{1}{\mathrm{~T}}\left|\int_{0}^{\mathrm{T}} w s s_{\mathrm{i}} d t\right|},
\end{array}
$$

where $w s s_{\mathrm{i}}$ is the instantaneous WSS vector and $T$ is the duration of the cycle. The OSI was averaged over the dome area.

\section{Statistical Analysis}

Statistical analyses were performed by using Excel 2003 (Microsoft, Redmond, Washington) and SAS 9.1 (SAS Institute, Cary, North Carolina). Hemodynamic parameters were normalized by the prestenting status values in each case to investigate the rate of variation caused by different stent placement strategies. Then the Kruskal-Wallis $H$ test was used, followed by the Nemenyi test for multiple comparisons. $P<.05$ (2-sided) was the criterion for statistical significance.

\section{RESULTS}

All the virtual stents were deployed successfully in the 10 VAFA models with the 3 different strategies. Their locations (beginning and end of the stent) were consistent with the realistic stents derived from the 2D DSA images (Fig 1).

As the number of stents increased, WSS was observed to decrease gradually by $7.2 \%, 20.6 \%$, and $25.8 \%$; and after the third stent was implanted, the variation reached a significantly different level compared with the prestenting status. Meanwhile, the RRT revealed progressive elevation by $11.3 \%, 15.4 \%$, and $45.0 \%$ and obtained a significant difference after the third stent used. The pressure on the aneurysmal wall increased by $15.7 \%, 21.5 \%$, and $28.2 \%$ as 3 stents were implanted sequentially. The variation rate of the pressure was significantly different from the prestenting status, even when only 1 stent was deployed. As to the OSI, the modification was revealed to be fluctuant, without any significant difference compared with the prestenting status (Fig 2). The median variation of each parameter is shown in Table 2. Further comparison of different stent placement strategies with the prestenting status is shown in Table 3.

Changes of the flow patterns before and after stent implantation were observed by plotting the speed vector field. The results showed that the flow patterns were not significantly changed after stent implantation. However, the intensities of the vortices were 


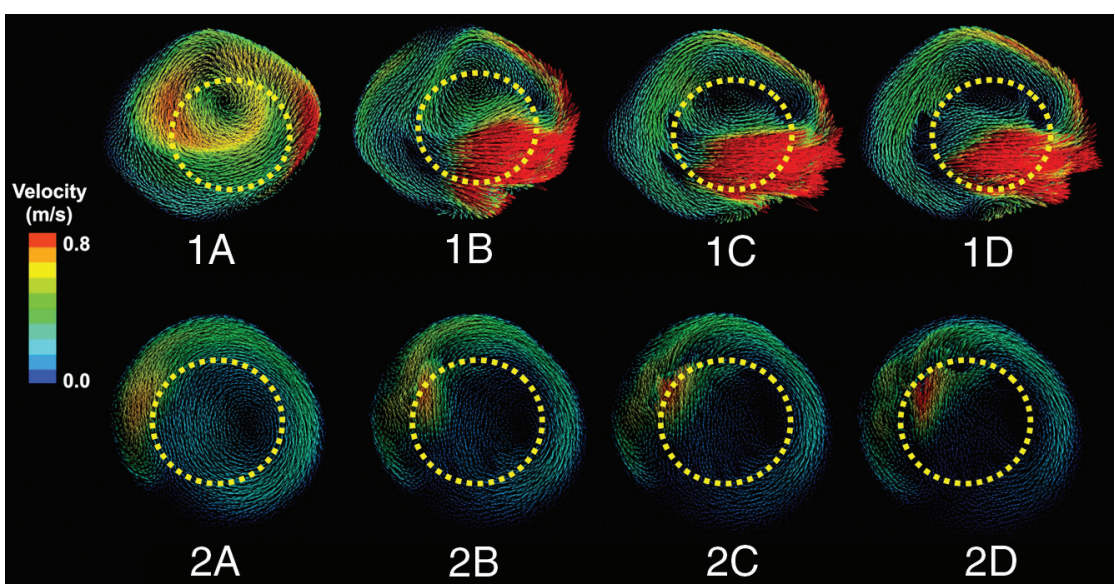

FIG 3. Flow patterns of 2 vertebral artery fusiform cases with different stent-placement strategies. One and 2 represent 2 individual cases. From left to right, flow patterns before and after implantation of 1-3 stents. The yellow dotted lines represent the positions of the stents. As the number of stents increased, blood flow inside the stent lumen concentrated and the flow rate accelerated, while the flow rate inside the aneurysmal sacs slowed and the vortex intensity weakened.

reduced, and the number of vortices in some cases was decreased (Fig 3).

\section{DISCUSSION}

Intracranial fusiform aneurysms are dilations of the parent artery without a clear aneurysmal neck. Similar to the widespread application of intracranial stent placement, endovascular treatment of fusiform aneurysms has been an important method and has greatly improved the long-term prognosis. ${ }^{8}$ This effect was even more significant when multiple overlapping stents were used. ${ }^{9,10}$ Although flow-diverter stents have been demonstrated to be an efficient treatment for aneurysms, their safety in posterior fusiform aneurysms still needs to be established by more evidence, especially because some important branches of smaller arteries might become covered. ${ }^{11}$ Therefore, stepwise overlapping stent placement is still a safe and feasible choice when dealing with posterior fusiform aneurysms.

In saccular aneurysm studies, both in vivo and in silico experiments have demonstrated that multiple stent implantations can reduce injective blood flow and create a hemodynamic environment, which will promote thrombus formation and finally cure the aneurysm. ${ }^{12}$ However, few hemodynamic studies have focused on intracranial fusiform aneurysms, in which hemodynamic patterns are different from those of saccular lesions. The application of the CFD technique makes hemodynamic studies in aneurysms more feasible and measurable. The fast virtual stent technique, first introduced by Larrabide et al, ${ }^{7}$ provides an accurate and efficient method to consider the behavior of stents in patient-specific aneurysm models. Being different from previous finite element analysis and porous medium methods, the fast virtual stent method shows a high consistency with real stents and an impressive efficiency. ${ }^{5,13}$ On the basis of this technique, we simulated the Enterprise stent and investigated the performance of different stent-placement strategies in 10 patient-specific VAFA models, aiming to obtain more evidence for clinical decision-making.

Thrombus formation in the aneurysm sac and consequent re- construction of the parent artery are the primary goals of stent placement in fusiform aneurysms. The stents should increase the time of blood flow staying in the aneurysm to create an environment for thrombosis, to achieve these goals. ${ }^{14}$ RRT is an important parameter that reflects the disturbed blood flow and its prolonged residence time in the aneurysmal sac. Tanemura et $\mathrm{al}^{6}$ demonstrated that a single Enterprise stent could increase the RRT by $20 \%$ in a previous CFD study. Our study further demonstrated that the RRT gradually increases by sequential stent placements, which would likely provide a better environment for thrombus formation.

WSS is the most studied hemodynamic parameter that has been demonstrated to play an important role in aneurysm initiation, rupture, and recanalization. ${ }^{15}$ Previous studies on saccular aneurysms suggested that stent implantation can lead to reduction of WSS and that this effect is related to the stent porosity-the lower the stent porosity, the more significantly the WSS decreased, and the distribution of WSS of the aneurysm became more uniform. ${ }^{16}$ In our study, progressive reduction of WSS was observed in VAFAs as the number of stents increased, which was similar to that in saccular aneurysms. However, lower WSS might also increase the risk of aneurysmal growth and even rupture, which has been shown in our previous CFD studies in saccular lesions. We therefore deduce that if thrombosis does not occur in a relatively short time, the aneurysms would be at risk of deterioration.

Another concern is the observed elevation of the pressure in every case and even for only a single stent. In a hemodynamic study of flow diverters by Cebral et al, ${ }^{17}$ a significant increase in pressure in some cases was observed after flow-diverter implantation, which, as those authors wrote, might lead to aneurysm rupture. However, some researchers had reported controversial results of a pressure decrease through virtual stent implantation in a fusiform aneurysm. ${ }^{18}$ These findings indicate that the modification of pressure may be related to patient-specific characteristics, which, in turn, might be related to the morphologic differences of aneurysms and stents. The average elevation of pressure in this study was $28.2 \%$. Coils might play a protective role in these VAFAs and should be considered, especially in cases in which CFD indicates a pressure increase.

The intra-aneurysmal flow pattern, reflecting the overall hemodynamic characteristics of an intracranial aneurysm, is reported to be more complex in ruptured aneurysms. ${ }^{17}$ Stent implantation might modify the complex flow pattern into a simplified pattern by reducing the power of vortices and secondary flows, which may create favorable conditions for intra-aneurysmal thrombosis. In the present study, most of the cases had $>2$ independent vortices before stent placement and the number of 
vortices changed during the cardiac cycle in the most complex situations. After stent deployment, weakening of the intensity of the vortices and shifting of the vortex center from the aneurysmal wall were observed in most cases, indicating an improvement of the flow pattern.

According to the results of multiple comparisons among different groups, single stents might not affect the hemodynamics as significantly as multiple stents. This possibility is consistent with clinical experience in that multiple stents improved the long-term results in VAFAs. However, as the number of stents increases, lower WSS and higher pressure might amplify the risk of rupture and recanalization; moreover, there was a greater theoretic chance for occlusion of some vital perforating branches. Although no hemorrhage or ischemic events were encountered in these patients, careful decision-making for the stent-placement strategy and timely follow-up are required.

There are several limitations in this study that might influence the results. First, because this is a patient-specific study and each case might have its own characteristics, the small sample size may be the main limitation. Second, the boundary conditions of the simulations were unified, and some perforating branches were neglected artificially. We did not consider potential changes of the inflow and outflow conditions, which might occur after stent placement. Changes of these boundary conditions will modify the absolute values of the investigated hemodynamic parameters. The observed trends however (ie, decrease in WSS, OSI, and RRT and an increase in pressure) are mostly driven by the separation of the hemodynamic environment of the aneurysmal sac from the parent artery and therefore should remain valid in a small or moderate variation of the boundary conditions. Absolute values derived from computational simulations should always be considered with caution and should be confirmed with actual measurements. In addition, the biologic process of thrombosis formation was too complex to simulate and was omitted.

\section{CONCLUSIONS}

The hemodynamic mechanism of endovascular stent placement in treating fusiform aneurysms can be explored with the virtual stent-placement technique. Stent placement modified hemodynamic patterns in VAFAs toward favoring thrombosis conditions in the aneurysmal sac. This effect was amplified with increasing numbers of stents. However, the potential risk of rupture or recanalization exists and should be considered when planning to use the multiple-stent technique in VAFAs.

Disclosures: Ignacio Larrabide-RELATED: Support for Travel to Meetings for the Study or Other Purposes: Agencia Nacional de Promoción Científica y Tecnológica, Comments: grant PICT 2015-0006*; UNRELATED: Employment: Consejo Nacional de Investigaciones Científicas Y Técnicas, Comments: Research Fellow. *Money paid to the institution.

\section{REFERENCES}

1. Drake CG, Peerless SJ. Giant fusiform intracranial aneurysms: review of 120 patients treated surgically from 1965 to 1992. J Neurosurg 1997;87:141-62 CrossRef Medline

2. Echiverri HC, Rubino FA, Gupta SR, et al. Fusiform aneurysm of the vertebrobasilar arterial system. Stroke 1989;20:1741-47 CrossRef Medline

3. Yoon W, Seo JJ, Kim TS, et al. Dissection of the V4 segment of the vertebral artery: clinicoradiologic manifestations and endovascular treatment. Eur Radiol 2007;17:983-93 CrossRef Medline

4. Siddiqui AH, Abla AA, Kan P, et al. Panacea or problem: flow diverters in the treatment of symptomatic large or giant fusiform vertebrobasilar aneurysms. J Neurosurg 2012;116:1258-66 CrossRef Medline

5. Augsburger L, Reymond P, Rufenacht DA, et al. Intracranial stents being modeled as a porous medium: flow simulation in stented cerebral aneurysms. Ann Biomed Eng 2011;39:850-63 CrossRef Medline

6. Tanemura H, Ishida F, Miura Y, et al. Changes in hemodynamics after placing intracranial stents. Neurol Med Chir (Tokyo) 2013;53: 171-78 CrossRef Medline

7. Larrabide I, Kim M, Augsburger L, et al. Fast virtual deployment of self-expandable stents: method and in vitro evaluation for intracranial aneurysmal stenting. Med Image Anal 2012;16:721-30 CrossRef Medline

8. Yang P, Zhao K, Zhou Y, et al. Stent-assisted coil placement for the treatment of 211 acutely ruptured wide-necked intracranial aneurysms: a single-center 11-year experience. Radiology 2015;276: 619 CrossRef Medline

9. Mehta B, Burke T, Kole M, et al. Stent-within-a-stent technique for the treatment of dissecting vertebral artery aneurysms. AJNR AmJ Neuroradiol 2003;24:1814-18 Medline

10. Won YS, Rho MH, Chung EC, et al. Multiple overlapping stentassisted coiling of complex aneurysms: a single-center experience. Neurol Res 2015;37:189-96 CrossRef Medline

11. Bhogal P, Pérez MA, Ganslandt O, et al. Treatment of posterior circulation non-saccular aneurysms with flow diverters: a single-center experience and review of 56 patients. J Neurointerv Surg 2017;9: 471-81 CrossRef Medline

12. Hashimoto $\mathrm{T}$, Meng H, Young WL. Intracranial aneurysms: links among inflammation, hemodynamics and vascular remodeling. Neurol Res 2006;28:372-80 CrossRef Medline

13. Thériault $P$, Terriault $P$, Brailovski V, et al. Finite element modeling of a progressively expanding shape memory stent. J Biomech 2006; 39:2837-44 CrossRef Medline

14. Cantón G, Levy DI, Lasheras JC, et al. Flow changes caused by the sequential placement of stents across the neck of sidewall cerebral aneurysms. J Neurosurg 2005;103:891-902 CrossRef Medline

15. Lv N, Wang C, Karmonik C, et al. Morphological and hemodynamic discriminators for rupture status in posterior communicating artery aneurysms. PLoS One 2016;11:e0149906 CrossRef Medline

16. Liou TM, Li YC. Effects of stent porosity on hemodynamics in a sidewall aneurysm model. J Biomech 2008;41:1174-83 CrossRef Medline

17. Cebral JR, Mut F, Raschi M, et al. Aneurysm rupture following treatment with flow-diverting stents: computational hemodynamics analysis of treatment. AJNR Am J Neuroradiol 2011;32:27-33 CrossRef Medline

18. Tremmel M, Xiang J, Natarajan SK, et al. Alteration of intra-aneurysmal hemodynamics for flow diversion using Enterprise and Vision stents. World Neurosurg 2010;74:306-15 CrossRef Medline 